\title{
Dust around Herbig Ae/Be stars: modelling of observational data
}

\author{
Natalia A. Krivova ${ }^{1,2,3}$, Vladimir B. Il'in ${ }^{2}$, and Hiroshi Kimura ${ }^{3}$ \\ ${ }^{1}$ Nicholaus Copernicus Astronomical Center, Warsaw, Poland \\ ${ }^{2}$ Astronomical Institute, St. Petersburg University, St. Petersburg, Russia \\ ${ }^{3}$ MPI für Aeronomie, Katlenburg-Lindau, Germany
}

(Received November 4, 1997; Revised January 14, 1998; Accepted January 14, 1998)

\begin{abstract}
Herbig Ae/Be (HAeBe) stars, young stars surrounded by dust shells, are believed to be precursors of $\beta$ Pic-like stars, and the dust around them is thought to be a possible source material for the formation of planets. A group of the HAeBe stars (UX Ori, WW Vul, etc.) shows large brightness variations. The dust in the vicinity of these stars is responsible not only for their excess emission in the infrared, anomalous extinction in the ultraviolet and visual, and specific spatial distributions of the intensity and polarization, but also for the "blueing" effect in the colour-magnitude diagrams and the intrinsic polarization increase observed in deep minima.

In contrast to the previous studies, we take advantage of a simultaneous modelling of all the observational data mentioned. Monte-Carlo simulations of polarized radiation transfer in the shells with a spheroidal density distribution have been performed for different dust grain models. The results are compared with observations of a typical HAeBe star WW Vul. Some effects related to a possible porosity of the circumstellar grains are considered.
\end{abstract}

\section{Introduction}

Herbig Ae/Be $(\mathrm{HAeBe})$ stars are young, pre-main sequence stars of moderate mass $\left(2-8 \mathrm{M}_{\odot}\right)$ with emission lines in the spectra and large infrared (IR) excesses which originate in the dusty shells. It is commonly believed that the HAeBe stars represent the early evolutionary stages of $\beta$ Pic-type stars and the dust around them could be a possible source material for the formation of planets. The circumstellar (CS) dust reveals itself in the observed IR excesses, anomalous $\mathrm{CS}$ extinction, intrinsic polarization of the stellar radiation and the specific spatial distributions of the intensity and polarization.

A numerous subgroup of UX Ori-like stars is marked by large (up to $2^{m}-4^{m}$ ) irregular variability which is interpreted as being due to their obscurations by dense CS clumps (Wenzel, 1969). In deep minima a blueing of the colour indices and an increase of the linear polarization degree have been observed many times (see Grinin, 1994 and references therein) and were attributed to the increasing role of the light scattered by small CS dust particles (Grinin, 1988).

Some of the observed phenomena (thermal emission of dust in the near and mid IR, scattering and polarization of the stellar radiation, CS extinction) should be caused by dust in the same, innermost layers of the shells. Therefore, it is reasonable to consider the phenomena simultaneously and to try to find a model satisfying all the observational data. However, previous studies have shown that it is already difficult enough to explain a part of the data (e.g. Sorrell, 1990; Grady et al., 1995; Krivova and Il'in, 1997). In addition, there exists the problem of the quick removal of any submicron grains out of the shells by radiation pressure (Voshchinnikov

Copy right $(\mathrm{C}$ The Society of Geomagnetism and Earth, Planetary and Space Sciences (SGEPSS); The Seismological Society of Japan; The Volcanological Society of Japan; The Geodetic Society of Japan; The Japanese Society for Planetary Sciences. and Grinin, 1991; Il'in and Voshchinnikov, 1993). It should be noted that similar difficulties are faced when studying $\beta$ Pic (see e.g. Nakamura, 1998), and the presence of porous, cometary-like grains around it has been inferred $(\mathrm{Li}$ and Greenberg, 1998).

In this paper, we make an attempt to model as many observational data on the dust shells surrounding the HAeBe stars with Algol-like brightness minima as possible. The effect of dust fluffiness on the results is considered in order to study the potentialities of the fluffy grain models to solve the problems indicated.

\section{Model}

We adopt the variable CS extinction model (Wenzel, 1969; Grinin, 1988), which means that the brightness dimmings are due to dense clumps moving around the star and screening it from an observer.

Our "spheroidal shell model" includes a sphericallyshaped shell of size $R_{\text {out }}$ with a spherical dust-free zone of radius $R_{\text {in }}$ due to dust sublimation and the density distribution

$$
n(\vec{r})=n_{0}^{\prime}\left[\sqrt{r_{x}^{2}+r_{y}^{2}+r_{z}^{2}(a / b)^{2}}\right]^{-\alpha}
$$

where $\left(r_{x}, r_{y}, r_{z}\right)$ are the coordinates of the radius-vector $\vec{r}$, $a / b$ is the oblateness of the spheroidal isodenses.

The following dust models have been considered:

(i) Mathis et al. (1977, MRN) model of compact bare silicate and graphite grains with the power-law size distribution

$$
n(a) \propto n^{-q},
$$

and the minimum and maximum sizes $a_{\min }$ and $a_{\max }$, respectively;

(ii) Mathis (1996) model with moderately porous, inhomogeneous grains (fluffy aggregates of silicate, amorphous 
carbon and graphite subparticles) with the size distribution

$$
n(a) \propto a^{-q} e^{-\left(\gamma_{1} a+\gamma_{2} a^{-1}+\gamma_{3} a^{2}\right)},
$$

where $\gamma_{1}, \gamma_{2}, \gamma_{3}$ are constants;

(iii) Li and Greenberg (1998) model with highly porous grains (aggregates of silicate core-organics mantle subparticles) with an exponential size distribution.

Fluffiness and inhomogeneity of the grains in the models were treated approximately by using an effective refractive index calculated with the Bruggeman rule (Bohren and Huffman, 1983).

Simulations of polarized radiation transfer were performed with a 2D Monte Carlo code (Fischer, 1995). The dust temperature distribution $T(r)$ was taken as that in a spherical shell with size $R_{\text {out }}$, inner hole radius $R_{\text {in }}$ and the density distribution

$$
n(r)=n_{0} r^{-\alpha},
$$

where $n_{0}$ was chosen so that the masses of the spheroidal and spherical shells were equal. The radiation transfer problem for the spherically symmetric shell model was treated with a 1D code of E. Krügel (Chini et al., 1986).

The changes of the colour indices $\Delta(X-Y)$ during stellar minima caused by eclipsing clumps were calculated from simple formula following Voshchinnikov and Grinin (1991).

\section{Results and Discussion}

We have calculated the spectral energy distribution from the UV to far IR, the stellar tracks on the colour-magnitude diagrams, the linear polarization at different brightness levels, and the spatial distributions of the intensity and polarization for several HAeBe stars, but here the results for only one star-WW Vul-are discussed.

WW Vul is a typical HAeBe star with Algol-like minima. It has the effective temperature $T_{*}=9500 \mathrm{~K}$, luminosity $L_{*}=80 L_{\odot}$, distance $d=550 \mathrm{pc}$, and the colour excess $E_{B-V}=0^{m} .345$ (Friedemann et al., 1993). IR observations of WW Vul revealed a considerable excess of the radiation (Cohen, 1973; Glass and Penston, 1974; Weaver and Jones, 1992; Li et al., 1994). The blueing effect on the colourmagnitude diagrams has been repeatedly noted in deep minima (Zaitseva, 1983; Berdyugin et al., 1992; Herbst et al., 1994). An increase of the linear polarization degree (up to $6 \%$ ) during several stellar brightness dimmings was observed by Berdyugin et al. (1992). It should be remarked that the observed polarization for stars with the shells can include the following components: the polarization caused by scattering of the stellar light in a non-spherical dust shell $\vec{P}_{\text {sca, }}$, the polarization due to optical dichroism of the CS dust $\vec{P}_{\mathrm{CS}}$, and the interstellar polarization $\vec{P}_{\text {IS }}$. Berdyugin et al. (1992) have estimated all the components for WW Vul. Our modelling can provide only $P_{\text {sca }}=\sqrt{Q^{2}+U^{2}} / I$, where $I, Q, U$ are the Stokes parameters of the shell radiation.

We begin our consideration with spherically symmetric shell models to find the dependence $T(r)$ and the values of the parameters that weakly depend on the (spheroidal) geometry of the shell. From fitting the spectral energy distribution in the visual and IR and the colour-magnitude diagrams for the UBVRI bands, we estimated: the inner $\left(R_{\text {in }} \approx 2 \mathrm{AU}\right)$ and outer $\left(R_{\text {out }} \approx 10^{4} \mathrm{AU}\right)$ radii of the shell, the power-law index of the radial density distribution $(\alpha \approx 1.5)$, the mass of the dust shell $\left(M_{\mathrm{env}} \approx 10^{-5} M_{\odot}\right)$ and its optical thicknesses for absorption $\left(\tau_{V}^{\mathrm{abs}} \approx 0.20\right)$ and scattering $\left(\tau_{V}^{\mathrm{sca}} \approx 0.08\right)$ in the visual (see Krivova, 1997 for details). Note, that such a modelling enables one to determine the shell parameters, but not the CS dust grain characteristics, except for the mean albedo at the wavelengths of about $0.3-0.5 \mu \mathrm{m}$ (Krivova and Il'in, 1997).

Based on these results, we have constructed spheroidal models of the shell for different dust grain models. Some results obtained with the Mathis (1996) dust model are presented in Fig. 1. The model allows us to fit the spectral energy distribution and the $P_{\text {sca }}(\lambda)$ dependence and gives a "flat" CS extinction curve in the UV as is usually observed for the HAeBe stars. However, the contribution of the scattered light to the total radiation of the object $(\mathrm{star}+$ shell) for it is too high, which is why the tracks on the colour-magnitude diagrams do not agree with the observations. The scattered light fraction depends on the angle $i$ between the shell symmetry axis and the line of sight as more radiation is scattered along the equatorial plane of the shell. Consequently, a reasonable decrease of $i$ (say, down to $60-70^{\circ}$ ) leads to some reduction of the fraction which, however, on the one hand, is still insufficient to explain the colour-magnitude diagrams (Fig. 1(c)), and on the other hand, is accompanied by a drop of the linear polarization degree $P_{\text {sca }}$ (Fig. 1(d)). It should be emphasized that we consider the spheroidal shell models with the oblateness $a / b=5$ which produces maximum polarization (see e.g. Voshchinnikov and Karjukin, 1994).

Let us now discuss the effects of possible fluffiness of the $\mathrm{CS}$ grains. The results of our calculations for the grains with the filling factor $f=0.15$ which is 3 times lower than that in the Mathis (1996) dust model $(f=0.45)$, are presented in Fig. 1. The porosity of the grains in the MRN model affects the results in a similar way and is not discussed here.

It is seen from Fig. 1 that for more fluffy particles, the near and mid-IR fluxes are slightly weaker, whereas the far-IR fluxes are slightly higher. However, the difference is of little significance. Therefore, the parameters of the shells such as the inner and outer radii, the power-law index of the radial density distribution, the optical thickness for absorption, etc. can be obtained from the modelling of IR spectra and spatial intensity distributions using compact grain models.

Another situation is with the mean albedo of the grains $\Lambda$. From the analysis of different observational data for WW Vul, Krivova and Il' in (1997) have obtained upper limits $\Lambda_{\mathrm{U}, \mathrm{B}, \mathrm{V}} \lesssim 0.3-0.4$ for the case when very small CS grains or/and PAH molecules do not strongly contribute to the near and mid-IR flux and the dust shell does not contain an optically thick disk. If the assumptions are correct, the value of $\Lambda$ for the CS dust grains is much lower than that typical of the interstellar clouds (about 0.5-0.7).

In the frame of the compact dust approach, this means that only small $(\leqq 0.05 \mu \mathrm{m})$ particles or small particles with a small addition of rather large $(\gtrsim 1 \mu \mathrm{m})$ grains are present in the shells. In this case, however, it is difficult to explain the large polarization degree and the rather flat CS extinction curve observed for UX Ori-like stars.

For porous particles, the albedo is smaller, and the grain models with a moderate porosity agree better with the colour- 

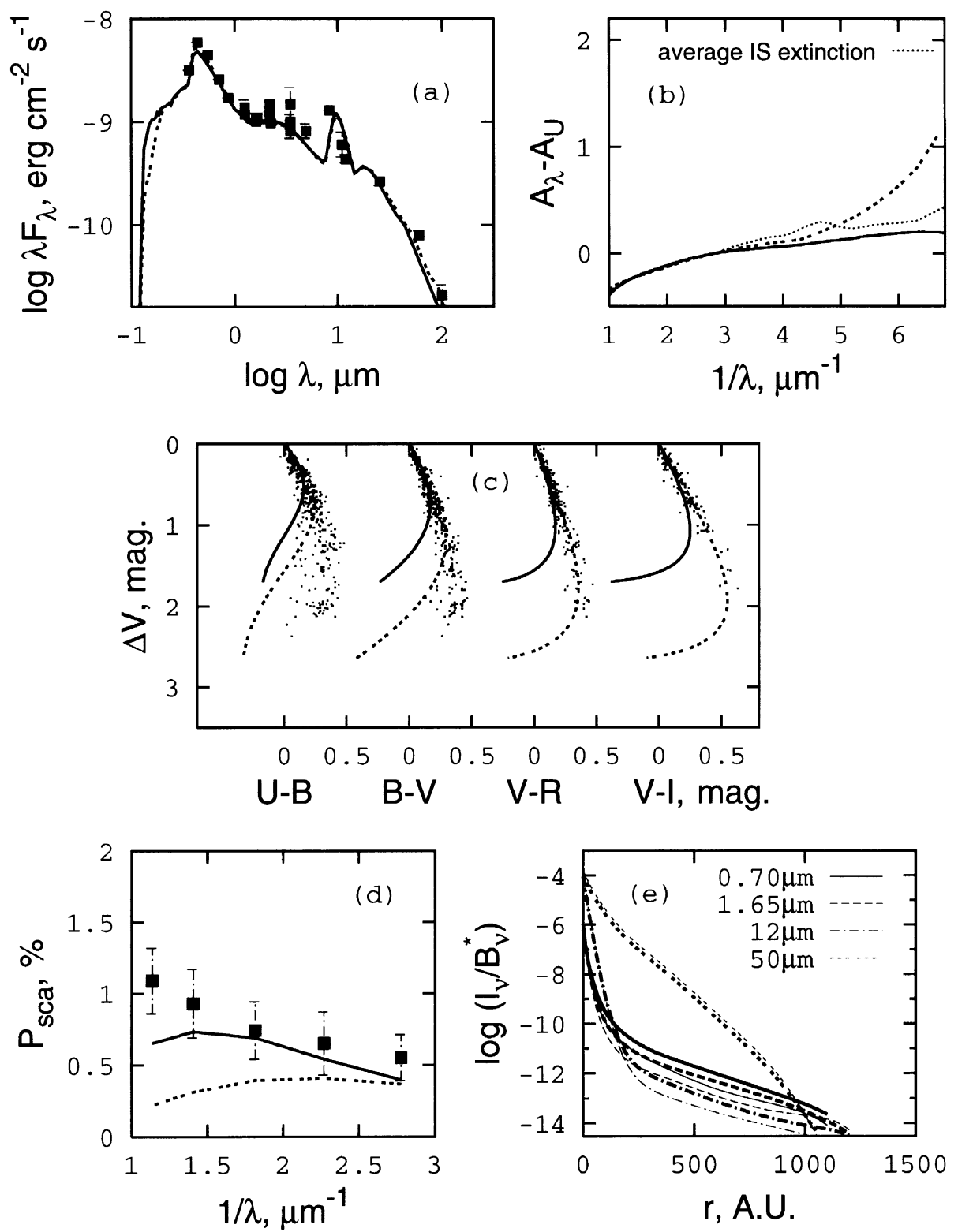

Fig. 1. The spectral energy distributions (a), the CS extinction curves (b), the colour-magnitude diagrams (c), the wavelength dependences of the intrinsic polarization (d), and the radial distributions of the specific intensity normalized to the stellar intensity (e) for a spheroidal model of the shell of WW Vul seen at the angle $i=70^{\circ}$. All plots except for (c) are given for the time of maximum brightness. The grain models with the filling factor $f=0.45$ (solid lines) and $f=0.15$ (dashed lines) have the averaged albedo $\Lambda_{\mathrm{U}}=0.59$ and $0.42, \Lambda_{\mathrm{V}}=0.56$ and 0.32 , respectively. Symbols are used for observational data. In (e) the results for $f=0.45$ and $f=0.15$ are shown with bold and thin lines, respectively; $r$ is the distance from the star in the plane perpendicular to the line of sight.

magnitude diagrams. For very fluffy particles, there is a steep rise of the CS extinction in the $\mathrm{UV}$, which is not observed for the HAeBe stars, and a decrease of the polarization degree in the visual down to quite low values. The radiation pressure is usually smaller for porous particles, but the decrease is not sufficient for them to stay long enough in the vicinity of these luminous stars.

It should be emphasized that in our modelling the grain porosity was treated by a reduction of the refractive index of spherical particles. Another approach could be sophisticated simulations of the optical properties of really fluffy aggregates using the Discrete Dipole Approximation (DDA). Our DDA calculations for ballistic particle-cluster and cluster- cluster aggregates have shown that the asymmetry factor of light scattering $g$ for the aggregates is larger (more light is scattered forward) than that for the spheres with the corresponding effective refractive index (see Kimura and Mann, 1998 for details). As results, the radiation pressure on the aggregates should be smaller than that on the compact spheres and the dependence of the scattered light fraction in the object radiation on the angle $i$ should be stronger. The rise of the single-scattering albedo with decreasing wavelength is less sharp for the aggregates than that for the spheres, and this fact should also reduce the problems appearing in the modelling of observations (Krivova and Il'in, 1997). As a next step, we plan to consider the optical properties of fluffy 
aggregates in more detail and to carry out radiative transfer calculations for the models involving the light scattering by the aggregates.

\section{Summary}

We have carried out Monte Carlo calculations of polarized radiation transfer in the shells with a spheroidal density distribution. Various observational data were simulated using several dust grain models and compared with those available for a typical HAeBe star WW Vul. The results obtained can be summarized as follows.

It is difficult to put strong constraints on the CS dust grain models, except, probably, for the mean albedo in the UBV bands. On the contrary, the parameters of the shells can be estimated relatively well.

From fitting the different observational data we found that the contribution of the scattered light to the total radiation of the object (star+shell) should be much lower than the amount we obtained with dust grains typical of the diffuse interstellar medium. This can be attributed to several possibilities: low albedo of the grains (e.g. due to their fluffiness), strong contribution (up to $50 \%$ ) of very small grains or/and $\mathrm{PAH}$ molecules to IR excess, or some geometrical effects.

If fluffy particles are present in the shells, the number of highly porous, cometary-like grains is rather limited. A moderate fluffiness of the CS grains better agrees with the observational data, but only slightly reduces the ratio of the radiation pressure force to the gravity force. So, the problem of the very fast removal of the grains remains in the scenario of continuous replenishment of CS dust by clumps (cometlike bodies) orbiting close to the stars (Voshchinnikov and Grinin, 1991; Li and Greenberg, 1998).

Acknowledgments. We are grateful to Drs. E. Krügel and O. Fischer for providing us the codes, to Dr. N. V. Voshchinnikov for constructive comments, to Dr. I. Mann for useful discussions and support of this work, to Dr. A. Natta for review of the paper and suggestions that made it clearer and to Prof. J. F. McKenzie for reading the manuscript and improvement the style. N.K. is thankful to Dr. R. Szczerba for offering nice working conditions during her stay in Poland and to Profs. S. Mukai and T. Mukai for their warm hospitality during the ZCS Workshop. V.I. acknowledges the support within the "Astronomy" program of the Russian federal government.

\section{References}

Berdyugin, A. V., V. P. Grinin, and N. Kh. Minikulov, The optical dichroism of the circumstellar dust and intrinsic polarization of WW Vul, Izv. Krym. Astrofiz. Obs., 86, 69-96, 1992.

Bohren, C. F. and D. R. Huffman, Absorption and Scattering of Light by Small Particles, 530pp., J. Wiley \& Sons, New York, 1983.
Chini, R., E. Krügel, and E. Kreysa, Dust emission spectra from star-forming regions, Astron. Astrophys., 167, 315-324, 1986.

Cohen, M., Infra-red observations of young stars-II. T Tauri stars and the Orion Population, Monthly Not. Roy. Astron. Soc., 161, 97-104, 1973.

Fischer, O., Polarization by interstellar dust-modelling and interpretation of polarization maps, Rev. Mod. Astr., 8, 103-124, 1995.

Friedemann, C., H.-G. Reimann, J. Gürtler, and V. Tóth, The cloudy circumstellar dust shell of WW-Vulpeculae revisited, Astron. Astrophys., 277, 184-194, 1993.

Glass, I. S. and M. V. Penston, An infrared survey of RW Aurigae stars, Monthly Not. Roy. Astron. Soc., 167, 237-249, 1974.

Grady, C. A., M. R. Pérez, P. S. Thé, et al., The $\beta$ Pictoris phenomenon among young stars. II. UV observations of the Herbig Ae star UX Orionis, Astron. Astrophys., 302, 472-482, 1995.

Grinin, V. P., On the blue emission visible during deep minima of young irregular variables, Astron. Let., 14, 27-28, 1988.

Grinin, V. P., Polarimetric activity of Herbig Ae/Be stars, in The Nature and Evolutionary Status of Herbig Ae/Be Stars, edited by P. S. Thé et al., ASP Conf. Ser. 62, pp. 63-70, 1994.

Herbst, W., D. K. Herbst, and E. J. Grossman, Catalogue of UBVRI photometry of T Tauri stars and analysis of the causes of their variability, Astron. J., 108, 1906-1923, 1994.

Il'in, V. B. and N. V. Voshchinnikov, Dynamics of dust particles in the shells of Herbig Ae-stars, Astron. Rep., 37, 362-367, 1993.

Kimura, H. and I. Mann, Radiation pressure cross section for fluffy porous aggregates, J. Quant. Spectr. Rad. Transf., 1998 (in press).

Krivova, N. A., Herbig Ae stars with Algol-like minima: modeling of the spectral energy distribution and of the behavior of colors at minima, Astron. Let., 23, 327-337, 1997.

Krivova, N. A. and V. B. Il'in, Dust shells around Herbig Ae/Be stars with Algol-like minima: Modeling of photometric observations, Astron. Let., 23, 791-798, 1997.

$\mathrm{Li}$, A. and J. M. Greenberg, A comet dust model for the $\beta$ Pictoris disk, Astron. Astrophys., 331, 291-313, 1998.

Li, W., N. J. Evans, II., P. M. Harvey et al., Near-infrared J, H, K imaging of Herbig Ae/Be stars, Astrophys. J., 433, 199-215, 1994.

Mathis, J. S., Dust models with tight abundance constraints, Astrophys. J., 472, 643-655, 1996.

Mathis, J. S., W. Rumpl, and K. H. Nordsieck, The size distribution of interstellar grains, Astrophys. J., 217, 425-433 (MRN), 1977.

Nakamura, R., Optical properties of dust aggregates in the disk of $\beta$ Pictoris, Earth Planets Space, 50, this issue, 587-593, 1998.

Sorrell, W. H., Constraints on astronomical silicate dust, Astrophys. J., 361, 150-154, 1990.

Voshchinnikov, N. V. and V. P. Grinin, Dust around young stars-model of envelope of the Ae Herbig star WW-Vulpeculae, Astrophysics, 34, 84, 1991.

Voshchinnikov, N. V. and V. V. Karjukin, Multiple scattering of polarized radiation in circumstellar dust shells, Astron. Astrophys., 288, 883-896, 1994.

Weaver, W. B. and G. Jones, A catalog of co-added IRAS fluxes of Orion population stars, Astrophys. J. Suppl. Ser., 78, 239-266, 1992.

Wenzel, W., in Non-Periodic Phenomena in Variable Stars, edited by L. Detre, p. 61, IAU Coll., Budapest, 1969.

Zaitseva, G. V., The photoelectric photometry of WW-Vulpeculae in 19671982, Perem. Zvezdy, 22, 1, 1983.

N. A. Krivova (e-mail: natalie@astro.spbu.ru), V. B. Il'in (e-mail: ilin@ astro.spbu.ru), and H. Kimura (e-mail: kimura@helene.mpae.gwdg.de) 